\title{
Quantitative sensory tests fairly reflect immediate effects of oxycodone in chronic low-back pain
}

\author{
Jürg Schliessbach ${ }^{\mathrm{a}, *}$, Andreas Siegenthaler ${ }^{\mathrm{b}}$, Lukas Bütikofer ${ }^{\mathrm{c}}$, Pascal Vuilleumier ${ }^{\mathrm{a}}$, \\ Peter Jüni ${ }^{\mathrm{d}}$, Lars Arendt-Nielsen ${ }^{\mathrm{e}}$, Michele Curatolo ${ }^{\mathrm{e}, \mathrm{f}}$ \\ a Department of Anesthesiology and Pain Therapy, Inselspital, Bern University Hospital, University of Bern, Switzerland \\ ${ }^{\mathrm{b}}$ Chronic Pain Management, Lindenhof Hospital, Lindenhof Group Bern, Switzerland \\ ${ }^{c}$ CTU Bern, and Institute of Social and Preventive Medicine (ISPM), University of Bern, Switzerland \\ ${ }^{\mathrm{d}}$ Applied Health Research Centre (AHRC), Li Ka Shing Knowledge Institute of St. Michael's Hospital, Department of Medicine, University of Toronto, Canada \\ e Centre of Sensory Motor Interaction SMI, School of Medicine, University of Aalborg, Denmark \\ ${ }^{\mathrm{f}}$ Department of Anesthesiology and Pain Medicine, University of Washington, Seattle WA, USA
}

H I G H L I G H T S

- Oxycodone had a significant analgesic effect on low-back pain compared to placebo.

- Oxycodone had significant anti-nociceptive effects on almost all QST modalities.

- Anti-nociceptive effects assessed by QST fairly reflect efficacy of oxycodone.

- QST may be more useful to identify non-responders rather than potential responders.

\section{A R T I C L E I N F O}

\section{Article history:}

Received 1 May 2017

Received in revised form 29 June 2017

Accepted 5 July 2017

\section{Keywords:}

Quantitative sensory tests

Chronic low-back pain

Oxycodone

Experimental pain

Analgesic effect

Anti-nociceptive effect

\begin{abstract}
A B S T R A C T
Introduction: Quantitative sensory tests (QST) can be used for profiling anti-nociceptive effects of analgesics. However, anti-nociceptive effects detected by QST are not necessarily associated with analgesic effects in pain patients. As part of a large investigation on low back pain, this paper describes the immediate analgesic and anti-nociceptive effects of oxycodone in chronic low-back pain and ranks different QST according to their ability to reflect this effect. The results are expected to support the selection of QST for future studies on potential novel opioid agonists in human pain.

Methods: In this randomized, placebo-controlled and double-blinded cross-over study, 50 patients with chronic low-back pain received a single oral dose of oxycodone $15 \mathrm{mg}$ or active placebo, and underwent multiple QST testing. The intensity of low-back pain was recorded during $2 \mathrm{~h}$. The areas under the ROC curves and $95 \%$ confidence intervals were determined, whereby responder status ( $\geq 30 \%$ pain reduction) was set as reference variable and changes in QST from baseline were set as classifiers.

Results: Significant analgesic effect on low-back pain as well as anti-nociceptive effects for almost all QST parameters were observed. The QST with the highest area under the curve were heat pain detection threshold $(0.65,95 \%$-CI 0.46 to 0.83$)$, single-stimulus electrical pain threshold $(0.64,95 \%$-CI 0.47 to 0.80$)$ and pressure pain detection threshold $(0.63,95 \%-\mathrm{CI} 0.48$ to 0.79$)$.

Conclusions: The results suggest that anti-nociceptive effects assessed by QST fairly reflect clinical efficacy of oxycodone on low-back pain. Pressure pain detection threshold, heat pain detection threshold and single-stimulus electrical pain threshold may be more suitable to sort out potential non-responders rather than identifying potential responders to opioid medication. Future pre-clinical human research may consider these results when investigating the analgesic effect of opioid agonists by means of QST.
\end{abstract}

\footnotetext{
* Corresponding author at: University Department of Anesthesiology and Pain Therapy, Inselspital, CH-3010 Bern, Switzerland. E-mail address: juerg.schliessbach@insel.ch (J. Schliessbach).
} 


\section{Introduction}

Opioid prescription for chronic pain has considerably increased in the past years, particularly for chronic low-back pain [1]. However, long-term use of opioids for non-cancer conditions is controversial because of numerous side effects such as development of tolerance, respiratory depression, constipation or drug misuse. A recent systematic review found opioids to be better than placebo, but not necessarily better than non-opioid analgesics for chronic low-back pain [2].

Most available studies that have examined the short-term effects of opioids did so by means of quantitative sensory tests (QST) in healthy volunteers [3-6]. However, a significant anti-nociceptive effect detected by QST in pain-free volunteers does not necessarily imply that the drug exerts a clinically meaningful analgesic effect in pain patients. Given the broad spectrum of QST modalities, it would be of relevance to know which QST parameter best reflects the clinical effect of a drug in a given patient population.

The present study is part of a larger project that investigated the ability of QST to predict the efficacy of several drugs in chronic lowback pain. This sub-study describes the immediate analgesic effect of oxycodone on chronic low-back pain and its anti-nociceptive effects as assessed by QST. The different QST are ranked according to their ability to reflect these effects. The results are expected to support the selection of QST for future studies on potential novel opioid agonists in human pain.

\section{Methods}

This was a randomized, double-blinded and placebo-controlled study at the University Department of Anesthesiology and Pain Medicine, Inselspital Bern, Switzerland. It was registered with clinicaltrials.gov (NCT01179828) and approved by the local ethics committee (KEK 213-09). The detailed study protocol [7] and results from another substudy [8] have been published. All participants gave written informed consent prior to enrolment.

\subsection{Patients}

Consecutive patients aged $18-80$ years with chronic low-back pain of at least 3 months duration were eligible. Pain intensity at the moment of testing had to be $\geq 3 / 10$ on the numerical rating scale (NRS, $0=$ no pain and $10=$ worst pain imaginable). Exclusion criteria were: suspected radicular pain (as defined by leg pain associated with an MRI finding of a herniated disc or spinal stenosis), signs or suspicion of neurological dysfunction at the tested sites, pregnancy (as assessed by pregnancy test), breast feeding, treatment with an antidepressant, opioid or anticonvulsant, intake of centrally active substances (including drug or alcohol abuse), allergy or pharmacological contraindications to the tested drugs, systemic inflammatory or rheumatologic disease, and major depression (Beck Depression Inventory short form score $>9$ ). Analgesic medication was stopped one week before the first experiment. Only acetaminophen or ibuprofen were allowed as rescue medication until $24 \mathrm{~h}$ before the experiment.

\subsection{Medication and measurements}

In this cross-over study, oxycodone $15 \mathrm{mg}$ and tolterodine $1 \mathrm{mg}$ (active placebo) were administered in a randomized, doubleblinded fashion, after $6 \mathrm{~h}$ of fasting and with a minimal wash-out time of 1 week between experiments. Tolterodine was chosen as an active placebo because it is a centrally active anti-cholinergic drug that mimics some of the side-effects of oxycodone (e.g. drowsiness, light sedation), but is devoid of any analgesic effect. Each the verum and the placebo pills were concealed by the hospital pharmacy using identical-looking red gelatin capsules and packed in semi-opaque plastic flasks labelled with subject number, lot number and expiry date. Neither the subject nor the investigators were aware which flask contained which substance. Randomization was performed by the pharmacist using a computer-generated random list.

\subsection{Quantitative sensory testing}

Quantitative sensory testing (QST) was performed at the leg and arm of the more painful body side (or randomly selected side in case of bilateral/midline pain). Pressure pain detection and tolerance thresholds (PPDT and PPTT) were measured at the second toe using an electronic algometer (Somedic AB, Horby, Sweden) with a probe surface of $1 \mathrm{~cm}^{2}$. Pressure was increased at a rate of $30 \mathrm{kPa} / \mathrm{s}$ until the sensation became painful (PPDT) or intolerable (PPTT).

Electrical single-stimulation pain threshold (ESPT) and electrical repeated-stimulation pain threshold (ERPT with 5 stimuli at $2 \mathrm{~Hz}$ inducing temporal summation) were measured using a constant current stimulator (Digitimer Ltd, Welwyn Garden City, UK) and two surface electrodes attached below the lateral malleolus. Bursts of five $1 \mathrm{~ms}$ square wave impulses within $25 \mathrm{~ms}$ (perceived as one single stimulus) were delivered with current intensity increasing by $0.5 \mathrm{~mA}$ until the sensation became painful (ESPT). For ERPT, the stimuli were repeated five times at a frequency of $2 \mathrm{~Hz}$. Current intensity of all 5 stimuli was increased in steps of $0.5 \mathrm{~mA}$ until the last 2-3 stimuli were perceived as painful, indicating temporal summation threshold.

Heat pain detection and tolerance thresholds (HPDT and HPTT) and cold pain detection threshold (CPDT) were measured at the leg (L5-dermatome) and at the forearm (C6-dermatome) using a thermode (TSA II, Medoc, Ramat Yishai, Israel). All measurements started at $30.0^{\circ} \mathrm{C}$, the rate of temperature change was $1{ }^{\circ} \mathrm{C} / \mathrm{s}$. Subjects stopped the measurements by pressing a button when the warm sensation turned to pain (HPDT) or when the pain became intolerable (HPTT) or when the cold sensation started to become painful (CPDT). In any case, the measurements were stopped at a temperature of $50.5^{\circ} \mathrm{C}$ for HPTT or $0^{\circ} \mathrm{C}$ for CPDT, respectively. CPDT was dichotomized into patients reaching $0^{\circ} \mathrm{C}$ without pain ("CPDT at limit") and patients who reported pain above $0^{\circ} \mathrm{C}$ ("CPDT not at limit").

Conditioned pain modulation (CPM) was assessed using the cold pressor test as conditioning stimulus. Electrical train-of-five stimulation was delivered at an intensity 1.2 times stronger than the previously determined ERPT. This was used as test stimulus and its painfulness was rated by the subjects on a 0-10 NRS. After this rating, subjects immersed their contralateral hand into an icesaturated water bath $\left(1.5 \pm 1^{\circ} \mathrm{C}\right)$. Once the cold pain reached an intensity of 7 on the $0-10 \mathrm{NRS}$, the test stimulus was repeated at the same current intensity. Again, a pain rating of that test stimulus was obtained. The time until cold pain reached $7 / 10$ was recorded. A decrease in pain rating of the test stimulus was considered a measure of CPM.

The normative values of all mentioned QST (except CPM) have been determined in a large sample of 300 healthy volunteers [9] and - with exception of CPDT - have also been shown to have acceptable test-retest reliability [10].

\subsection{Outcome measures}

Low-back pain intensity $2 \mathrm{~h}$ after drug intake was the primary outcome measure. NRS scores for pain in the supine and sitting position were recorded at baseline and in intervals of $30 \mathrm{~min}$ for up to $2 \mathrm{~h}$ after drug intake. Drug responders were defined as patients having $\geq 30 \%$ pain reduction after $120 \mathrm{~min}$. The patients' global 
impression of change scale (PGIC) was assessed on a 7 point scale ranging from " $1=$ very much improved" over " $4=$ no change" to "7 = very much worse", in intervals of $30 \mathrm{~min}$, starting $30 \mathrm{~min}$ after drug administration.

Age, sex, body mass index (BMI), pain duration in years, history of surgery due to the painful condition, average pain intensity during the last $24 \mathrm{~h}$ on a $0-10 \mathrm{NRS}$, pain-related life interference from the multidimensional pain inventory (MPI), catastrophizing scale and Beck Depression Inventory (BDI) were recorded as descriptive variables.

\subsection{Statistical analyses}

\subsubsection{Analgesic and anti-nociceptive effects}

Continuous and ordinal variables that were roughly normally distributed (NRS, PGIC, and CPM) or normally distributed after logtransformation (PPDT, PPTT, ESPT, and ERPT) were analyzed by linear mixed models with treatment group, time point and their interaction as covariates. The models were adjusted for baseline values and treatment phase (oxycodone first vs. placebo first) in order to account for a possible learning effect. A carry-over effect was excluded by design (wash-out period between the phases) and was not tested for. A random intercept was added for each subject (to account for intra-subject correlation) and a random intercept and slope for each subject in each treatment phase (to account for repeated measures). Correlations between subsequent measurements were modelled with a first order autoregressive correlation structure. The treatment effect was calculated over all time points (joint $p$-value) and at each time point based on appropriate contrasts and is presented as mean difference or geometric mean ratio (if data was log-transformed). Dichotomous variables (drug responders, dichotomized CPDT) were modelled by separate logistic generalized estimating equations (GEEs) at each time point with treatment group and phase as covariates and subject ID as panel variable and the treatment effect is presented as odds ratio. HPDT and HPTT which were truncated at $50.5^{\circ} \mathrm{C}$ were analyzed by separate mixed tobit regression models at each time point with treatment group and phase as covariates and subject ID as panel variable. The treatment effect is presented as mean difference. Sample size calculation was performed for the outcome of the main project, i.e. for the ability of baseline QST to predict the analgesic effect of the tested drug after $2 \mathrm{~h}$. Detailed considerations can be found in the study protocol [7].

\subsubsection{Receiver-operating characteristics (ROC)}

The ability of QST to reflect drug effect was assessed by receiveroperating characteristic (ROC) analysis. For this purpose, only the results of the verum session were considered. Drug responder status in supine and sitting position $2 \mathrm{~h}$ after drug intake was used as reference variable to classify patients into "positive" and "negative" state, respectively. The changes in QST after $2 \mathrm{~h}$ were expressed relative to baseline and used as classifying variables. The area under the curve (AUC) and the 95\% confidence intervals were calculated by non-parametric ROC-analysis for the following QST parameters: PPDT, PPTT, ESPT, ERPT, HPDT at leg and arm, and time until cold pressor pain reached NRS 7/10. Heat pain tolerance and cold pain detection thresholds were not considered for ROC-analysis because of the truncation at $50.5^{\circ} \mathrm{C}$ and $0^{\circ} \mathrm{C}$, which was already reached at baseline by a substantial number of patients. For the purpose of a sensitivity analysis, ROC AUCs were as well calculated for the situations when drug responders were defined as having $\geq 20 \%$ and $\geq 40 \%$ pain reduction. All analyses were performed using STATA (STATA Corp, College station, Texas, USA).
Table 1

Descriptive variables.

$\begin{array}{ll}\text { Number of patients } & 50 \\ \text { Age } & 55.0 \pm 15.2 \\ \text { Sex (female) } & 26(52 \%) \\ \text { BMI }\left(\mathrm{kg} / \mathrm{m}^{2}\right) & 26.5 \pm 4.3 \\ \text { Pain duration (years) } & 12.3 \pm 11.1 \\ \text { Surgery due to pain (yes) } & 10(20 \%) \\ \text { Average NRS } & 4.9 \pm 1.4 \\ \text { Impairment of daily life } & 2.4 \pm 1.2 \\ \text { Catastrophizing score } & 1.6 \pm 1.3 \\ \text { Beck Depression Index } & 2.2 \pm 2.4\end{array}$

Descriptive variables and characteristics of the 50 patients included. BMI = body mass index, NRS $=$ pain intensity on numeric rating scale $(0=$ no pain, $10=$ worst pain imaginable). Data are presented as mean \pm SD or $n(\%)$.

Table 2

Baseline quantitative sensory tests.

\begin{tabular}{lll}
\hline & Verum session & Placebo session \\
\hline PPDT $(\mathrm{kPa})$ & $298(120)$ & $311(116)$ \\
PPTT $(\mathrm{kPa})$ & $465(151)$ & $480(140)$ \\
ESPT $(\mathrm{mA})$ & $6.4(3.4)$ & $7.0(3.7)$ \\
ERPT $(\mathrm{mA})$ & $5.4(2.8)$ & $5.7(3.1)$ \\
Iwsec $(\mathrm{s})$ & $23(30)$ & $23(29)$ \\
HPDT $\operatorname{arm}\left({ }^{\circ} \mathrm{C}\right)$ & $43.6(4.7)$ & $45.1(4.1)$ \\
HPDT leg $\left({ }^{\circ} \mathrm{C}\right)$ & $46.6(3.1)$ & $47.0(3.3)$ \\
HPTT $\operatorname{arm}\left({ }^{\circ} \mathrm{C}\right)$ & $48.8(2.2)$ & $49.1(2.01)$ \\
HPTT leg $\left({ }^{\circ} \mathrm{C}\right)$ & $49.6(1.3)$ & $49.7(1.4)$ \\
$\mathrm{CPDT} \operatorname{arm}\left({ }^{\circ} \mathrm{C}\right)$ & $11.9(11.3)$ & $11.9(10.6)$ \\
CPDT leg $\left({ }^{\circ} \mathrm{C}\right)$ & $8.0(10.8)$ & $8.4(10.5)$
\end{tabular}

Baseline values of quantitative sensory tests for both verum and placebo session. Values are presented as mean (SD). PPDT and PPTT = pressure pain detection and tolerance thresholds, ESPT and ERPT=electric single and repeated stimulus pain thresholds, Iwsec $=$ time in ice water in seconds until cold pain reached 7 on a $0-10$ numeric rating scale, HPDT and HPTT = heat pain detection and tolerance thresholds, $\mathrm{CPDT}=$ cold pain detection thresholds .

\section{Results}

Fifty patients were enrolled and all of them completed both the verum and the placebo session. There were 26 females and 24 males with a mean age of 55 years (SD 15.2). Patient characteristics and descriptive variables are shown in Table 1. Baseline values of QST are shown in Table 2.

\subsection{Low-back pain and QST}

A significant analgesic effect compared to placebo occurred after $30 \mathrm{~min}$ in the sitting and after $1 \mathrm{~h}$ in the supine position and remained throughout the rest of the observation period. Supine pain was 3.7 (95\%-CI 3.4 to 4.1 ) at baseline and decreased to 1.5 (1.1 to 2.0) with oxycodone and to 3.0 (2.4 to 3.5 ) with placebo after $2 \mathrm{~h}(p<0.001)$. Sitting pain was $4.2(3.9$ to 4.5$)$ at baseline and decreased to 1.6 (1.2 to 2.1 ) with oxycodone and to 2.9 (2.3 to 3.4) with placebo after $2 \mathrm{~h}(p<0.001)$. Time course of low-back pain and proportion of responders are shown in Fig. 1. Significant anti-nociceptive effects were observed for all QST except HPDT at the leg after $120 \mathrm{~min}$. CPM was at no time significantly different between oxycodone and placebo. PGIC was between 3 ("minimal improvement") and 4 ("no change) in both arms with a significant difference between treatments after 60 min only (see Table 3 for detailed results of QST and treatment effects).

\subsection{ROC-analyses}

For pain in the supine position, there were 36 responders vs. 13 non-responders. One patient was unable to rate his pain in the supine position (but had NRS $>3 / 10$ in sitting position), ROCanalysis for supine pain was therefore conducted on $n=49$ patients. 
Supine position
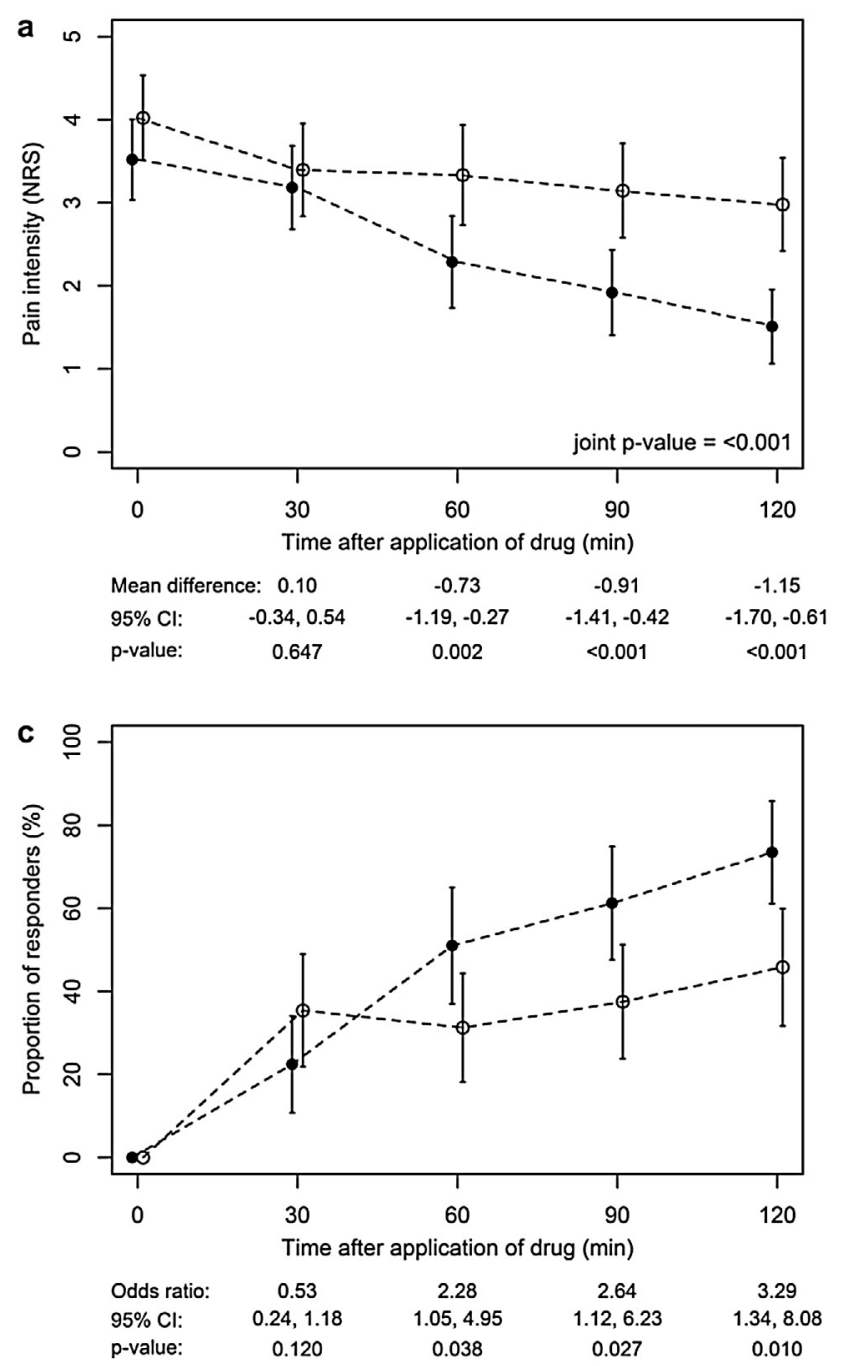

Sitting position Oxycodone $\rightarrow$ Placebo
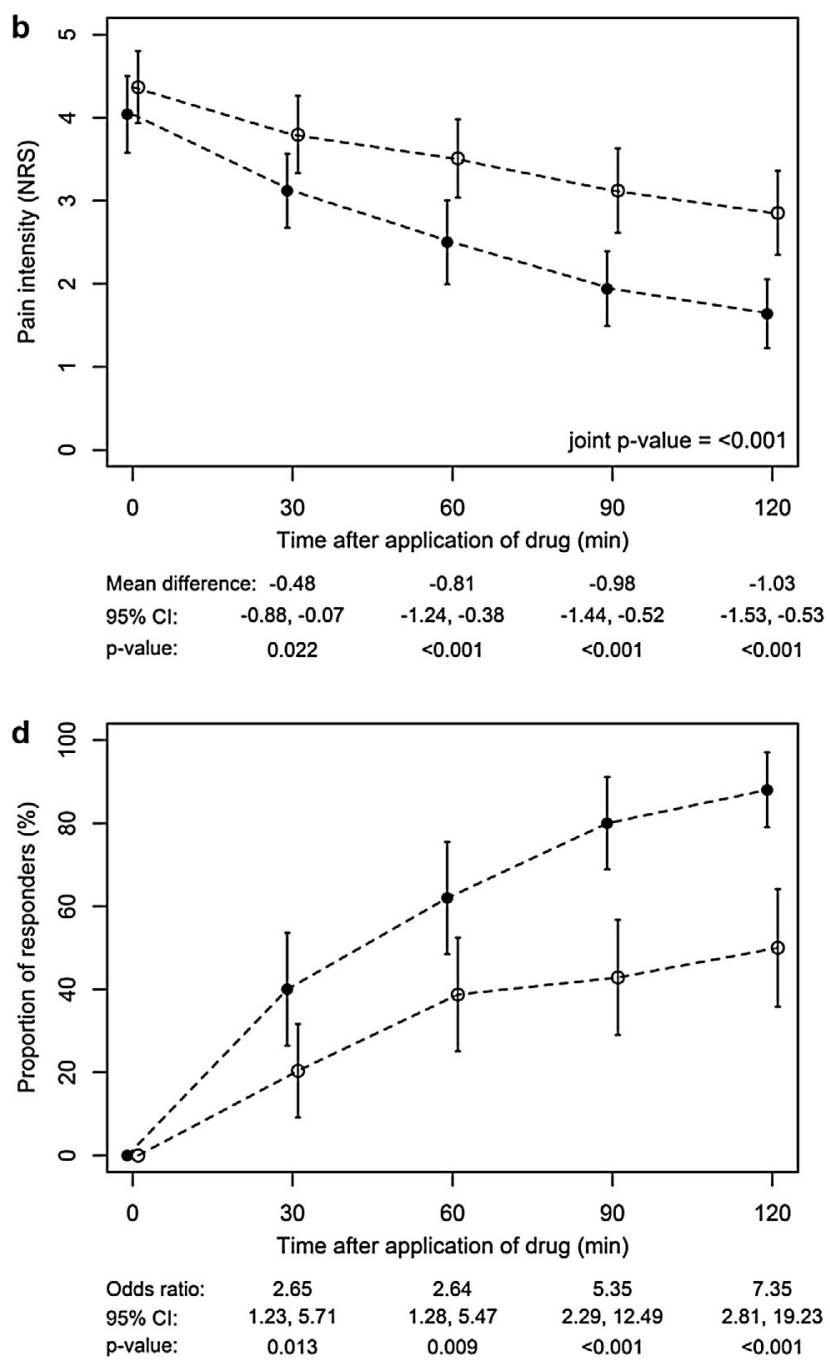

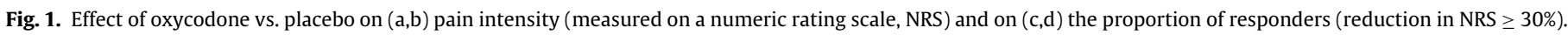
Pain intensity was measured in $(a, c)$ supine or $(b, d)$ sitting position. Values represent means and $95 \%$ confidence intervals.

The QST with the highest AUC were HPDT at the leg (AUC 0.65, $95 \%-\mathrm{CI} 0.46$ to 0.83$)$, ESPT $(0.64,0.47$ to 0.80$)$ and PPDT $(0.63,0.48$ to 0.79 ). For pain in the sitting position, there were 44 responders vs. 6 non-responders. Again, HPDT at the leg and ESPT showed the highest areas under the ROC-curve with 0.63 (0.35 to 0.90) for HPDT and 0.63 (0.42 to 0.83 ) for ESPT.

The sensitivity analysis with drug responders set as $\geq 20 \%$ pain reduction showed almost uniformly smaller AUCs. On the other hand, when drug responders were defined as $\geq 40 \%$ pain reduction, AUCs did not or only minimally increase compared to $\geq 30 \%$ pain reduction. Detailed results and 95\%-CIs are displayed in Table 4, ROC curves for the supine and sitting position are displayed in Figs. 2 and 3.

\subsection{Sensitivity and specificity}

As an example, sensitivity and specificity were calculated for those QST parameters with the highest AUC in supine position, i.e. PPDT, ESPT and HPDT at the leg. The chosen cutoffs were 1.11-fold and 1.2-fold increase from baseline for PPDT, 1.12-fold and 1.2fold increase from baseline for ESPT and 1.006-fold and 1.02-fold increase for HPDT. This choice was based on the experience of the authors, i.e. when measuring PPDT or ESPT, a change in thresholds by $10-20 \%$ is frequently observed, whereas for HPDT, changes of $0.5-1{ }^{\circ} \mathrm{C}$ (i.e. $1-2 \%$ increase) are far more realistic. Table 5 shows the values of sensitivity and specificity for each QST parameter and its respective cutoffs. Again, when the definition of responders was changed to $\geq 20 \%$ or $\geq 40 \%$ pain reduction, sensitivity and specificity were lower than with the definition of $\geq 30 \%$ pain reduction. In general, sensitivity was modest for the selected QST parameters, the most sensitive being a $12 \%$-increase in ESPT yielding a $75 \%$ sensitivity to identify a drug responder. However, specificity was $80-90 \%$ for PPDT and HPDT, indicating that these tests are more useful to rule out non-responders, rather than ruling in responders.

\section{Discussion}

\subsection{Analgesic and anti-nociceptive effects in relation}

This study showed that a single oral dose of oxycodone $15 \mathrm{mg}$ exerts a significant and immediate analgesic effect in chronic low-back pain which significantly differs from placebo. The effect occurred within $30 \mathrm{~min}$ in sitting position, but took slightly longer when patients were supine. As a possible explanation, opioids may 
Table 3

effect of oxycodone on quantitative sensory tests.

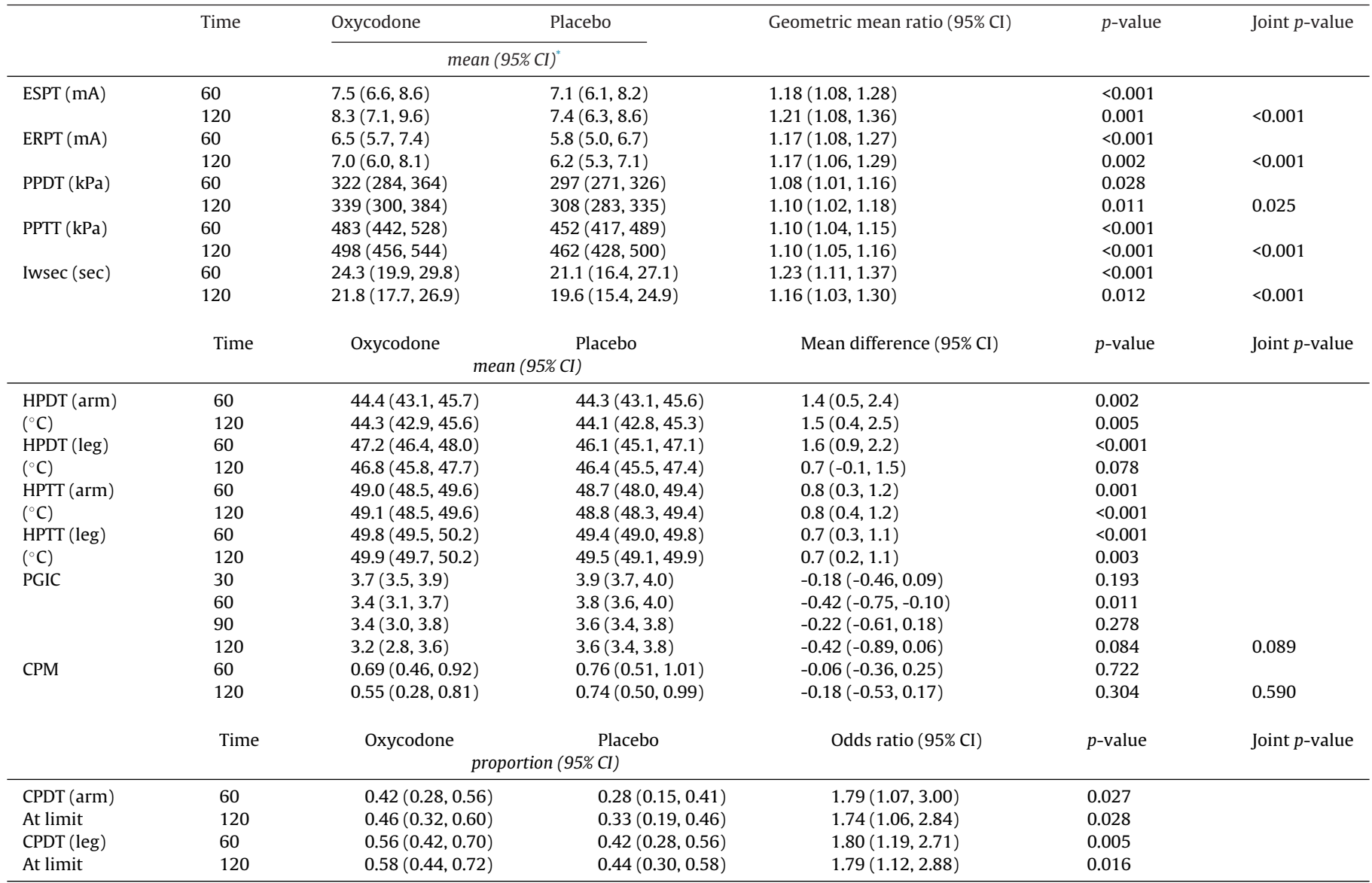

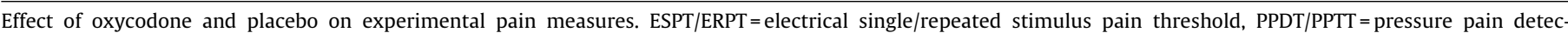

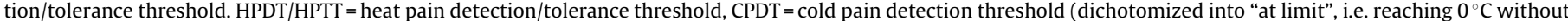

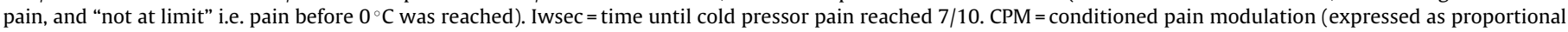
change from baseline). PGIC = patients' global impression of change scale (1-7 Likert-scale, 1 = very much improved, 7 = very much worse).

* calculated according to Cox's direct method.

Table 4

areas under the receiver-operating characteristics curve.

\begin{tabular}{|c|c|c|c|c|}
\hline & \multirow[t]{2}{*}{$N$} & \multicolumn{3}{|c|}{ ROC AUC for $x \%$ pain reduction $(95 \% \mathrm{CI})$} \\
\hline & & a) $x=20 \%$ & b) $x=30 \%$ & c) $x=40 \%$ \\
\hline \multicolumn{5}{|l|}{ Supine position } \\
\hline Ice time & 49 & $0.54(0.30,0.77)$ & $0.62(0.44,0.79)$ & $0.62(0.46,0.79)$ \\
\hline PPDT & 49 & $0.54(0.35,0.73)$ & $0.63(0.48,0.79)$ & $0.52(0.35,0.69)$ \\
\hline PPTT & 49 & $0.38(0.17,0.60)$ & $0.49(0.30,0.67)$ & $0.51(0.34,0.67)$ \\
\hline ESPT & 49 & $0.54(0.34,0.74)$ & $0.64(0.47,0.80)$ & $0.61(0.45,0.77)$ \\
\hline ERPT & 49 & $0.52(0.34,0.70)$ & $0.58(0.43,0.74)$ & $0.59(0.43,0.75)$ \\
\hline HPDT leg & 49 & $0.51(0.26,0.76)$ & $0.65(0.46,0.83)$ & $0.55(0.38,0.71)$ \\
\hline HPDT arm & $47^{*}$ & $0.72(0.53,0.92)$ & $0.63(0.43,0.83)$ & $0.63(0.47,0.80)$ \\
\hline \multicolumn{5}{|l|}{ Sitting position } \\
\hline Ice time & 50 & $0.46(0.21,0.71)$ & $0.48(0.26,0.70)$ & $0.50(0.30,0.69)$ \\
\hline PPDT & 50 & $0.50(0.22,0.78)$ & $0.51(0.27,0.75)$ & $0.56(0.37,0.74)$ \\
\hline PPTT & 50 & $0.49(0.25,0.73)$ & $0.53(0.32,0.74)$ & $0.64(0.47,0.82)$ \\
\hline ESPT & 50 & $0.57(0.35,0.79)$ & $0.63(0.42,0.83)$ & $0.66(0.49,0.84)$ \\
\hline ERPT & 50 & $0.43(0.18,0.68)$ & $0.46(0.24,0.68)$ & $0.60(0.44,0.77)$ \\
\hline HPDT leg & 50 & $0.58(0.26,0.89)$ & $0.63(0.35,0.90)$ & $0.64(0.46,0.82)$ \\
\hline HPDT arm & $48^{*}$ & $0.56(0.25,0.86)$ & $0.47(0.18,0.77)$ & $0.61(0.41,0.80)$ \\
\hline
\end{tabular}

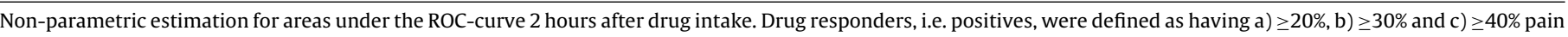

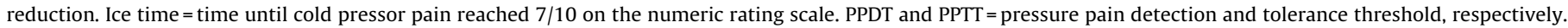
ESPT and ERPT = electrical single and repeated stimulus pain threshold, respectively. HPDT = heat pain detection threshold.

* Two patients had no thermal pain testing due to thermode malfunction. 

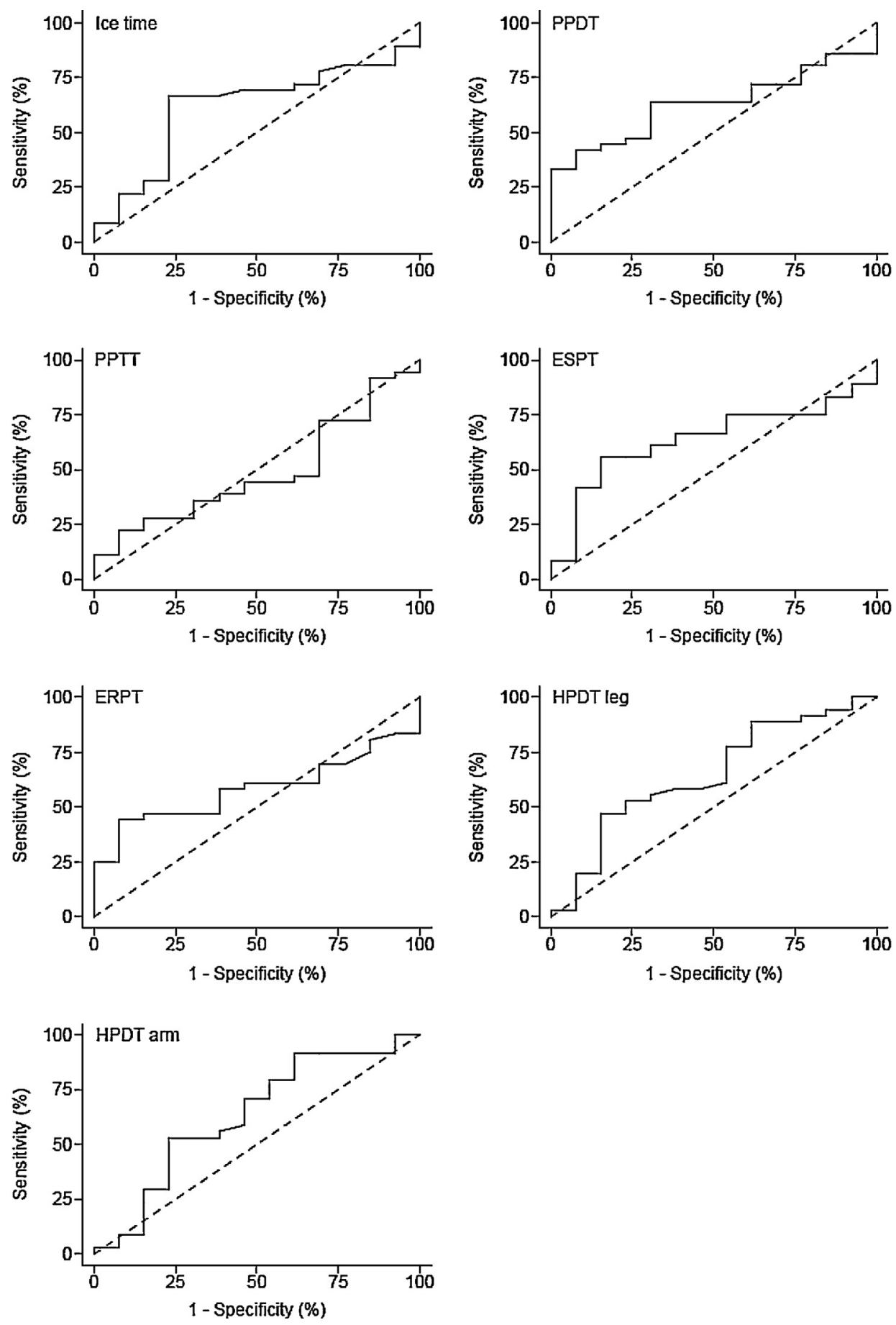

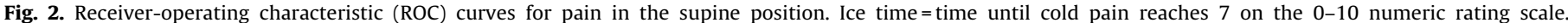
$\mathrm{PPDT} / \mathrm{PPTT}=$ Pressure pain detection/tolerance threshold, ESPT/ERPT $=$ electric single/repeated stimulus pain threshold, HPDT $=$ heat pain detection threshold.

be particularly useful to suppress peak nociceptive signals, as may result from mechanical strain or injury, while they are less efficient in blocking baseline nociceptive activity. The study simultaneously confirmed that oxycodone has anti-nociceptive effects on almost all sensory modalities, i.e. mechanical, electrical and thermal, and that they were affected in a manner similar to that observed in healthy volunteers [3-6].

Given the results from such studies, it might be tempting to infer that these anti-nociceptive effects translate into clinical analgesic effects in pain patients. However, not all QST reflected oxycodone effect on back pain to the same extent. For both supine and sitting pain, HPDT at the leg and ESPT consistently showed the highest areas under the ROC curve, with the maximal AUC of 0.65 observed for HPDT at the leg in supine position. This indicates that HPDT at the leg can correctly classify two randomly selected patients as a responder and non-responder in only $65 \%$ of the cases. Notably, the lower border of confidence intervals for all parameters is below 0.5 , which means that they might not be significantly better than a random classifier. Conversely, the upper border of confidence intervals barely exceeded 0.8 (except for HPDT at the leg), which implies that even under optimistic assumptions, QST could correctly classify responders and non-responders in no more than $\approx 80 \%$ of the 

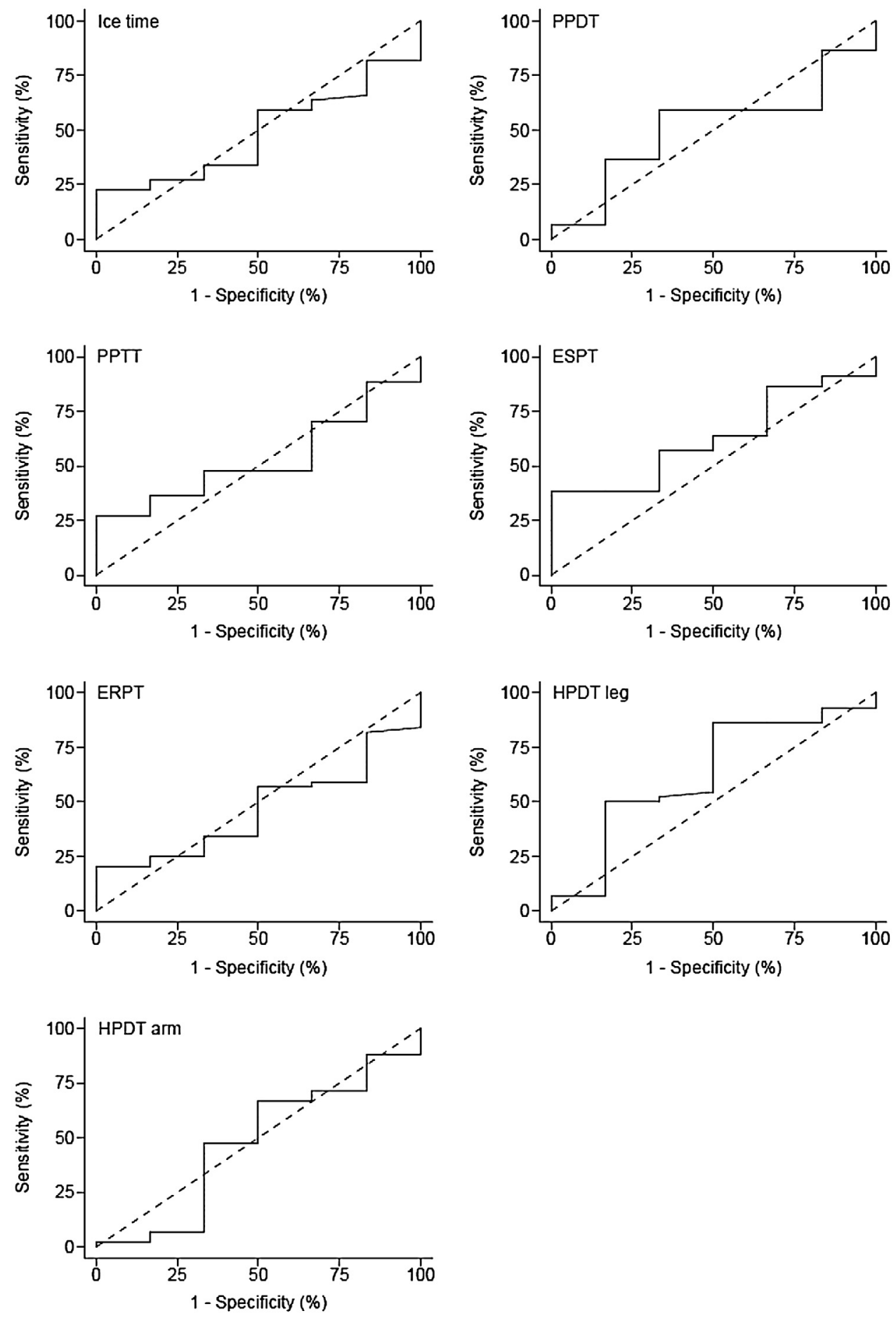

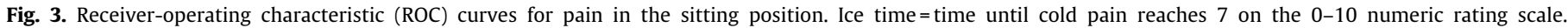
PPDT/PPTT = Pressure pain detection/tolerance threshold, ESPT/ERPT = electric single/repeated stimulus pain threshold, HPDT $=$ heat pain detection threshold.

cases. In the context of an approved drug like oxycodone, a $50-80 \%$ rate of correct classification by QST is modest. However, in the context of a pre-clinical investigation of a novel substance, which is tested first in volunteers before being used in pain patients, such information might still be useful.

PPDT for pain in the supine position had an AUC of 0.63 (95\%CI 0.48 to 0.79 ). This parameter might therefore correctly classify two randomly selected patients in $63 \%$ of the cases. In the sitting position, however, PPDT was not as good a classifier. The most probable explanation for the differences in AUC between sitting and supine position is the different proportion of responders and non-responders. In supine position, the ratio of responders to nonresponders was 36:13, whereas in sitting position, it was 44:6. This clearly limits the results of the ROC-analysis for the latter condition.

\subsection{Sensitivity analyses}

These results are contingent for a pain reduction $\geq 30 \%$ in patients who responded to oxycodone. In order to investigate how the ROC AUCs are influenced by this definition of responders, ROC 
Table 5

sensitivity and specificity of selected cutoff values of sensory tests.

\begin{tabular}{|c|c|c|c|c|c|c|}
\hline & & Cutoff & Sensitivity & Specificity & LR+ & LR- \\
\hline \multirow{6}{*}{$20 \%$ pain reduction } & \multirow[t]{2}{*}{ PPDT } & 1.11 & $51 \%$ & $67 \%$ & 1.5 & 0.7 \\
\hline & & 1.20 & $36 \%$ & $83 \%$ & 2.3 & 0.7 \\
\hline & \multirow[t]{2}{*}{ ESPT } & 1.12 & $73 \%$ & $17 \%$ & 0.9 & 1.6 \\
\hline & & 1.20 & $56 \%$ & $33 \%$ & 0.8 & 1.3 \\
\hline & \multirow[t]{2}{*}{ HPDT leg } & 1.006 & $41 \%$ & $67 \%$ & 1.2 & 0.9 \\
\hline & & 1.02 & $34 \%$ & $83 \%$ & 2.0 & 0.8 \\
\hline \multirow{6}{*}{$30 \%$ pain reduction } & \multirow[t]{2}{*}{ PPDT } & 1.11 & $58 \%$ & $82 \%$ & 3.2 & 0.5 \\
\hline & & 1.20 & $44 \%$ & $91 \%$ & 4.9 & 0.6 \\
\hline & \multirow[t]{2}{*}{ ESPT } & 1.12 & $75 \%$ & $27 \%$ & 1.0 & 0.9 \\
\hline & & 1.20 & $61 \%$ & $55 \%$ & 1.3 & 0.7 \\
\hline & \multirow{2}{*}{ HPDT leg } & 1.006 & $47 \%$ & $82 \%$ & 2.6 & 0.6 \\
\hline & & 1.02 & $39 \%$ & $91 \%$ & 4.3 & 0.7 \\
\hline \multirow{6}{*}{$40 \%$ pain reduction } & \multirow[t]{2}{*}{ PPDT } & 1.11 & $56 \%$ & $67 \%$ & 1.6 & 0.6 \\
\hline & & 1.20 & $43 \%$ & $80 \%$ & 2.2 & 0.7 \\
\hline & \multirow{2}{*}{ ESPT } & 1.12 & $75 \%$ & $27 \%$ & 1.0 & 0.9 \\
\hline & & 1.20 & $62 \%$ & $53 \%$ & 1.3 & 0.7 \\
\hline & \multirow[t]{2}{*}{ HPDT leg } & 1.006 & $47 \%$ & $73 \%$ & 1.7 & 0.7 \\
\hline & & 1.02 & $38 \%$ & $80 \%$ & 1.9 & 0.8 \\
\hline
\end{tabular}

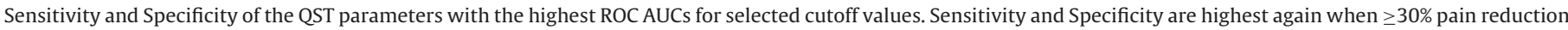

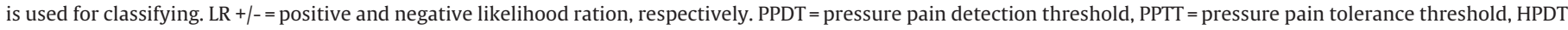
leg $=$ heat pain detection threshold measured at the leg (L5-dermatome).

analyses were carried out using $\geq 20 \%$ and $\geq 40 \%$ pain reduction as alternative definitions of drug responders. In the former cases, the AUCs were consistently lower, whereas in the latter they did not noticeably increase. A decrease in pain by $\geq 30 \%$ is often considered clinically meaningful, and the present results support the choice of this cutoff from a mechanistic, QST-based point of view. Concerning the sensitivity and specificity of QST, it is difficult to define a cutoff. In general, drug responders showed a broad spectrum of increases and decreases in QST parameters, whereas oxycodone non-responders showed barely any increase in QST. This is reflected by the rather modest sensitivity, but quite acceptable specificity of the QST parameters in the present analyses. Of course, the exact values of sensitivity and specificity depend on the cutoff one chooses, and the present results argue in favour of a cutoff that allows for a high specificity.

Among all recent studies investigating the effect of oxycodone, only one study combined the approach of using QST in chronic pain patients [11]. They found significant anti-nociceptive effects, especially on mechanical (i.e. skin pinch, muscle pressure) and heat pain tests in patients with chronic pancreatitis. Unfortunately, they did not report about the clinical effects of oxycodone on pancreatitis pain, so that no conclusion can be drawn whether these anti-nociceptive effects reflected clinical improvement as well.

\subsection{Strengths and limitations}

The study is novel in the sense that it combines both clinical and experimental pain measures in a chronic pain population. As this study was part of a larger investigation on low-back pain, sample size calculation was made for the primary aim of the project. There was no a priori sample size calculation for the ROC analysis, which is a limitation to this study. The relatively wide confidence intervals of the ROC AUCs might be an indication of lacking power. Yet, a sample of 50 patients should allow for reasonable estimation of effect size and a larger sample would probably change the width of the confidence intervals in the first line, rather than the effect size.

\section{Conclusions}

This study suggests that anti-nociceptive effects of oxycodone, measured by QST, reflect clinical efficacy to varying degrees. This should be considered when interpreting QST results from studies in pain-free volunteers. The QST parameters included in this study show at best a modest association. Heat pain detection threshold, single-stimulus electrical pain threshold and pressure pain detection threshold, may be more suitable classifiers, in particular to sort out non-responders, rather than to identify potential responders to the drug. Future pre-clinical human research may consider these findings when investigating the analgesic effect of opioid agonists by means of QST.

\section{Ethical issues}

This study was approved by the local ethics committee (KEK 213-09) and strictly followed the GCP guidelines and Helsinki declaration. All participants gave written informed consent prior to enrolment. The study was registered with clinicaltrials.gov (NCT01179828).

\section{Acknowledgements}

This study was supported by the Swiss National Science Foundation (SNF) in the context of the Special Program for University Medicine SPUM 33CM30_140339. The authors declare that they have no conflict of interests.

\section{References}

[1] Deyo RA, Von Korff M, Duhrkoop D. Opioids for low back pain. BM] 2015;350:g6380.

[2] Chaparro LE, Furlan AD, Deshpande A, Mailis-Gagnon A, Atlas S, Turk DC. Opioids compared to placebo or other treatments for chronic low-back pain. Cochrane Database Syst Rev 2013;8:CD004959.

[3] Zwisler ST, Enggaard TP, Noehr-Jensen L, Mikkelsen S, Verstuyft C, Becquemont L, Sindrup SH, Brosen K. The antinociceptive effect and adverse drug reactions of oxycodone in human experimental pain in relation to genetic variations in the OPRM1 and ABCB1 genes. Fundam Clin Pharmacol 2010;24:517-24.

[4] Staahl C, Christrup LL, Andersen SD, Arendt-Nielsen L, Drewes AM. A comparative study of oxycodone and morphine in a multi-modal, tissue-differentiated experimental pain model. Pain 2006;123:28-36.

[5] Olesen AE, Upton R, Foster DJ, Staahl C, Christrup LL, Arendt-Nielsen L, Drewes AM. A pharmacokinetic and pharmacodynamic study of oral oxycodone in a human experimental pain model of hyperalgesia. Clin Pharmacokinet 2010;49:817-27.

[6] Suzan E, Midbari A, Treister R, Haddad M, Pud D, Eisenberg E. Oxycodone alters temporal summation but not conditioned pain modulation: preclinical findings and possible relations to mechanisms of opioid analgesia. Pain 2013;154:1413-8. 
[7] Siegenthaler A, Schliessbach J, Vuilleumier PH, Juni P, Zeilhofer HU, ArendtNielsen L, Curatolo M. Linking altered central pain processing and genetic polymorphism to drug efficacy in chronic low back pain. BMC Pharmacol Toxicol 2015;16:23.

[8] Schliessbach J, Vuilleumier PH, Siegenthaler A, Butikofer L, Limacher A, Juni P, Zeilhofer HU, Arendt-Nielsen L, Curatolo M. Analgesic effect of clobazam in chronic low-back pain but not in experimentally induced pain. Eur J Pain 2017.

[9] Neziri AY, Scaramozzino P, Andersen OK, Dickenson AH, Arendt-Nielsen L, Curatolo M. Reference values of mechanical and thermal pain tests in a pain-free population. Eur J Pain 2011;15:376-83.
[10] Vuilleumier PH, Biurrun Manresa JA, Ghamri Y, Mlekusch S, Siegenthaler A, Arendt-Nielsen L, Curatolo M. Reliability of quantitative sensory tests in a low back pain population. Reg Anesth Pain Med 2015;40: $665-73$.

[11] Staahl C, Dimcevski G, Andersen SD, Thorsgaard N, Christrup LL, ArendtNielsen L, Drewes AM. Differential effect of opioids in patients with chronic pancreatitis: an experimental pain study. Scand J Gastroenterol 2007;42: 383-90. 